\title{
BMJ Open Dehydroepiandrosterone (DHEA) role in enhancement and maintenance of implantation (DREAM): randomised double-blind placebo-controlled trial - study protocol
}

\author{
Majiyd Abdul Noushin, ${ }^{1}$ Apeksha Sahu (D , , Swati Singh, ${ }^{1}$ Sankalp Singh, ${ }^{1}$ \\ Kannamannadiar Jayaprakasan, ${ }^{2,3}$ Reema Basheer, ${ }^{1}$ Aravind Ramachandran, ${ }^{1}$ \\ Mohamed Ashraf ${ }^{1}$
}

To cite: Noushin MA, Sahu A, Singh S, et al. Dehydroepiandrosterone (DHEA) role in enhancement and maintenance of implantation (DREAM): randomised doubleblind placebo-controlled trial一study protocol. BMJ Open 2021;11:e054251. doi:10.1136/ bmjopen-2021-054251

- Prepublication history for this paper is available online. To view these files, please visit the journal online (http://dx.doi. org/10.1136/bmjopen-2021054251).

Received 07 June 2021 Accepted 12 0ctober 2021

Check for updates

(c) Author(s) (or their employer(s)) 2021. Re-use permitted under CC BY-NC. No commercial re-use. See rights and permissions. Published by BMJ.

${ }^{1}$ Reproductive Medicine, CRAFT Hospital, Trichur, Kerala, India ${ }^{2}$ Nottingham University Research and Treatment Unit in Reproduction (NURTURE), University of Nottingham, Derby, UK

${ }^{3}$ Derby Fertility Unit, Royal Derby Hospital, Derby, UK

Correspondence to

Dr Apeksha Sahu;

drapekshargkar@gmail.com

\section{ABSTRACT}

Introduction Dehydroepiandrosterone (DHEA) is an important precursor of androgen and has been studied and researched extensively for improving the various outcome measures of ovarian stimulation in women with advanced age or poor ovarian response. Androgens also play an important role in the enhancement of endometrial and decidual function by regulating both the transcriptome and secretome of the endometrial stromal cells and have a positive effect on various factors like insulin-like growth factor binding protein 1, homeobox genes (HOXA10, HOXA11), secreted phosphoprotein 1 , prolactin which are necessary for implantation. It is well-known that the circulating 'precursor pool' of DHEA declines with age more so in poor ovarian reserve patients and exogenous supplementation may be beneficial in such cases. This double-blinded randomised controlled trial (RCT) aims to test the hypothesis whether transient targeted supplementation of DHEA as an adjuvant to progesterone in frozen embryo transfer (FET) cycles, for women with low serum testosterone, helps in improving live birth rate.

Methods and analysis This study is planned as a double-blinded, placebo-controlled randomised trial and the sample size, calculated for the primary outcome measure-live birth rate, is 140 . All participants will be having a flexible antagonist protocol for controlled ovarian stimulation and an elective freeze-all policy for the embryos as per the hospital protocol after written informed consent. For FET, the endometrium will be prepared by hormone replacement treatment protocol. During the FET cycle, the intervention group will be receiving DHEA $25 \mathrm{mg}$ two times a day for 15 days from the day of starting progesterone supplementation and the control group will be receiving a placebo.

Ethics and dissemination The approval of the study was granted by the Clinical Trials Registry-India and the Institutional Ethical Committee of CRAFT Hospital and Research Center. All participants will provide written informed consent before being randomised into allocated treatment groups. The results will be disseminated to doctors and patients through conference presentations,
Strengths and limitations of this study

- This is a double-blinded placebo-controlled randomised trial with a computer-generated system for randomisation that eliminates the possible bias.

- Evaluating the novel concept of the role of dehydroepiandrosterone (DHEA) in the luteal phase of the frozen embryo transfer (FET) cycle.

- The sample size and power calculation were focused on the primary outcome of the study, with limited power to detect other secondary outcome measures.

- The study intervention is limited to the hormone replacement treatment cycle, whether the same can be extrapolated to poor ovarian reserve patients undergoing natural cycle/downregulated FET cycle need to be examined.

- We did not assess serum DHEA levels as we expected serum DHEA to be low when serum testosterone is low and acknowledge this as a limitation.

peer-reviewed publications, social media and patient information booklets.

Trial registration numbers CTRI/2020/06/025918; ECR/1044/Inst/KL/2018.

\section{INTRODUCTION}

The establishment of a successful pregnancy depends on the embryo-endometrium cross-talk. For the endometrium to become receptive, endometrial stromal cells must undergo various functional and morphological differentiation to transform the decidual lining which acts as a bed for the embryo, the entire process is referred to as the process of decidualization. ${ }^{1}$ It is a complex process with the expression of various genes including HOXA10, HOXA11, FOXO1, ${ }^{2}$ WNT4, insulin-like growth factor binding protein 1 
$(\text { IGFBP-1 })^{3}$ and upregulation of progesterone, ${ }^{4}$ glucocorticoid, mineralocorticoid and androgen ${ }^{5}$ receptors in the endometrium. ${ }^{6}$

The androgen precursor, dehydroepiandrosterone (DHEA) has been studied and researched extensively for improving the various outcome measures of ovarian stimulation in women with advanced age or poor ovarian response. Though several meta-analyses ${ }^{78}$ showed beneficial effects of DHEA in women with diminished ovarian reserve, its potential role in enhancing decidualization is not explored well. The study by Gibson et al demonstrated that DHEA enhanced the expression and secretion of IGFBP-1, prolactin and secreted phosphoprotein 1 proteins and augmented the decidualization process with possible increased expression of progesterone receptors in vitro. ${ }^{9}$ It is well known that both homeobox genes (HOXA-10 and HOXA-11) and leukaemiainhibitory factor play a key role in the decidualization and implantation process ${ }^{10-12}$ and recent evidence shows upregulation of HOXA-10, HOXA-11 and leukaemiainhibitory factor with DHEA supplementation. ${ }^{6}$ DHEA supplementation also increased vascular endothelial growth factor-mediated angiogenesis which may enhance decidualization. ${ }^{13-15}$

With the increasing age, there is a decline in the synthesis of androgens from adrenal glands and ovary leading to a progressive fall in the level of androgens including local endometrial androgen and their metabolites. ${ }^{16}$ This is more so evident in poor ovarian reserve patients. ${ }^{17}$ These fascinating findings prompted us to evaluate the role of transient targeted supplementation of DHEA as an adjuvant to progesterone to improve the reproductive outcome in the frozen embryo transfer (FET) cycle, for women with low serum testosterone. To the best of our knowledge, there are no published in vivo studies on this aspect and the principal objective of this randomized controlled trial (RCT) is to evaluate whether DHEA supplementation during the luteal phase of the FET cycle improves the live birth rates.

\section{MATERIALS AND METHODS}

\section{Design, setting and participants}

This study is planned as a double-blinded, placebocontrolled randomised trial in an academic tertiary fertility centre (CRAFT Hospital and Research Centre, Kerala, India) after due approval from Institutional Ethics Committee (IEC) and prospective registration in Clinical Trial Registry-India. Patient recruitment started on 17 June 2020; tentative completion of enrolment will be by December 2021 and the result analysis will be by September 2022. During the FET cycle, the intervention group will be receiving DHEA $25 \mathrm{mg}$ two times a day for 15 days from the day of starting progesterone supplementation and the control group will be receiving a placebo with packaging, appearance and dosing similar to the intervention drug.
Unlike studies on DHEA for improving follicular recruitment, oocyte quality and quantity which required supplementation of 3-4 months, DHEA to enhance decidualization needed only limited days of supplementation. ${ }^{9}$ Taking into consideration that we did not want any premature decidualization process before we initiate progesterone, we decided to supplement DHEA only when we started progesterone and decided to offer it for 15 days arbitrarily based on our pilot study and as per the linear progression association put forth by Gibson $e t$ $a l .{ }^{9}$ The impact of DHEA on the expression of decidualization markers was also dose-dependent eliciting a bellshaped dose-response and the greatest increase in the expression of decidualization markers was detected after supplementation of DHEA in the physiological range. ${ }^{9}$ On account of this and the fact that both low and high levels of testosterone are detrimental for implantation ${ }^{1819}$ and endometrium can convert DHEA to active androgens and physiologically testosterone production is increased in the secretory phase ${ }^{20}$ we chose an arbitrary dosage of $50 \mathrm{mg}$ per day.

\section{Patient's recruitment randomisation}

In the FET cycle, once the endometrium is $\geq 8 \mathrm{~mm}$, patients will be assessed for the possibilities of inclusion for the trial which includes any one of the three:

1 . Women with age $\geq 35$ years or

2. Those with anti mullerian hormone (AMH) value less than $1.1 \mathrm{ng} / \mathrm{mL}$ (Beckman Coulter assay) or

3. Antral follicle count (AFC) less than 7 (ie, potential poor ovarian reserve patients).

These potential patients will be advised to give an early morning blood sample for serum testosterone and if their serum testosterone level is less than $0.2 \mathrm{ng} / \mathrm{mL}$, they will be considered for the trial.

\section{Exclusion criteria}

1. Women with polycystic ovarian syndrome (PCOS)/ clinical hyperandrogenaemia.

2. Women with serum testosterone $\geq 0.2 \mathrm{ng} / \mathrm{mL}$.

3 . Women with body mass index more than $30 \mathrm{~kg} / \mathrm{m}^{2}$.

4. Women with congenital and untreated or acquired uterine abnormality.

5. Women with untreated hydrosalpinx.

6. Women with thrombophilia.

7. Women with uncontrolled endocrine and haematological dysfunction.

8. Women undergoing preimplantation genetic testing and endometrial receptivity array testing.

9. Women undergoing natural or ovulation induction FET cycle/down-regulated FET cycle.

10. Women with thin endometrium less than $8 \mathrm{~mm}$ in the index cycle.

11. Severe male factor infertility.

12. Difficult embryo transfer cases (failed embryo transfer due to difficulty in negotiating external/internal cervical os due to pinpoint cervix, cervical stenosis or fibrosed cervical crypts). 


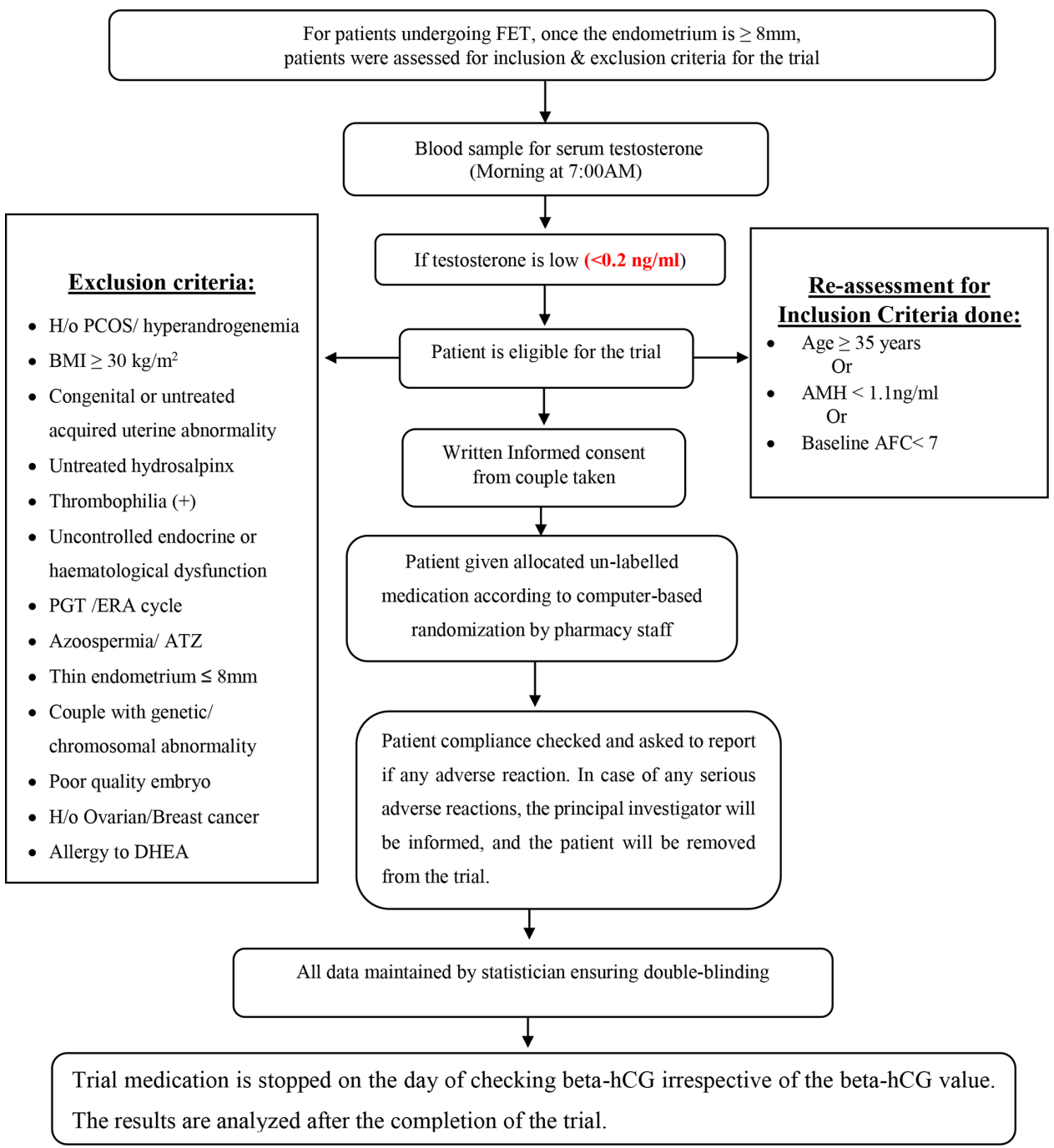

Figure 1 Patient flow chart: attached as a supplementary data. FET, frozen embryo transfer; PCOS, polycystic ovarian syndrome; PGT, preimplantation genetic treatment; ERA, endometrial receptivity array; ATZ, absolute teratozoospermia; BMI, body mass index; DHEA, dehydroepiandrosterone; beta-hCG, human chorionic gonadotropin; AMH, anti mullerian hormone; AFC, antral follicle count.

13. Cases where only poor-quality embryos are available for transfer.

14. Couple with genetic and chromosomal abnormality.

15. Known allergy to DHEA.

After fulfilling the inclusion and exclusion criteria, study participants are selected. Treating consultant will explain the study process to the patients and all participants willing for the study will provide written informed consent with signatures before randomisation into allocated treatment groups (figure 1). The randomisation will be performed by statistician through computergenerated random block sequencing; eligible patients will be randomly assigned to either study or control groups in a 1:1 ratio with random block sizes of four, six or eight subjects to prevent predictability of allocation. Both study participants and researchers/treating doctors will be blinded for the study (double-blinded study). Through computer-generated randomisation, patients will be given a unique identification number. The corresponding treatment allocation to this unique number will be transmitted to the pharmacy staff, who will dispense unlabelled strip containing capsules of either active $25 \mathrm{mg}$ DHEA (study group) or an inert ingredient (placebocontrol group) to be taken orally two times a day on the starting day of progesterone for the luteal phase support (LPS). Complete data of patient and treatment records will be documented in the electronic medical system database. All the pregnant patients will be followed till their pregnancy outcome is obtained which could be either delivery or miscarriage. Any adverse reaction or side effect reported by the women will be recorded and escalated to the principal investigator. Patients will be allowed to withdraw from the study anytime. Patient participation in the study can also be interrupted if the patient's general condition contradicts participation or there is a protocol violation that could influence the outcome measures.

\section{Primary objective}

The study aims to test the hypothesis whether the reproductive outcome is improved by androgen supplementation 
in the form of DHEA along with progesterone in FET cycles, in women with low serum testosterone, which may be either due to age-related changes or due to diminished ovarian reserve. The primary outcome measure is the live birth rate (defined as the delivery of a live-born $>24$ weeks of gestation) per embryo transfer cycle.

\section{Secondary objectives}

The secondary outcomes are pregnancy rate (serum betahuman chorionic gonadotropin (hCG) positive 2 weeks postembryo transfer), clinical pregnancy rate (evidence of intrauterine gestational sac with cardiac activity of the fetus at around 6-8 weeks' gestation) and miscarriage rate (defined as the proportion of women with pregnancy loss before 20 weeks of gestation) per embryo transfer cycle.

\section{Procedure}

All participants in the study and the control group will be having a flexible antagonist protocol for controlled ovarian stimulation and an elective freeze-all policy for the embryos as per the hospital protocol after written informed consent. For FET, the endometrium will be prepared by hormone replacement treatment protocol. Estradiol valerate (Progynova; Bayer Schering Pharma) is started on day 2 or 3 of the cycle at a dose of $2 \mathrm{mg}$ three times a day orally and subsequently, the dose will be stepped up as per the response of the endometrium to a maximum dose of $12 \mathrm{mg}$ per day. Follow-up transvaginal scan will be done to assess endometrial thickness from day 9 of the treatment cycle. Once the endometrium is $>8 \mathrm{~mm}$, as per the hospital standard operating procedure, before initiating progesterone for LPS we check serum progesterone to rule out escape ovulation and if patients fit into the potential study criteria serum testosterone is sent along with it. After randomisation patient will receive either an unlabelled strip of DHEA $25 \mathrm{mg}$ (study group) or a placebo (control group) to be taken orally two times a day on the day of starting of vaginal natural micronised progesterone (Cap Susten $400 \mathrm{mg}$ Sun Pharma) $400 \mathrm{mg}$ two times per day and oral dydrogesterone (Duphaston-Abbott India Ltd) $10 \mathrm{mg}$ three times a day for LPS. On the fourth day of progesterone supplementation, two embryos of day 3 developmental stage with at least one morphologically good quality or grade 1 embryo are transferred. Patients are excluded from the trial if the endometrial thickness is $<8 \mathrm{~mm}$ during the FET preparation or if there are no grade 1 (good)-quality embryos available for embryo transfer. All the embryos will be cultured till day 3 and will be graded as grade 1 (good) having $<10 \%$ fragmentation, cell number $>7$ on day 3 with no multinucleation. Grade 2 (fair) embryos refer to grade 1 embryos that have $10 \%-25 \%$ fragmentation. Embryos are tagged as grade 3 when cell number is $<7$ on day 3 or there is $>25 \%$ fragmentation or evidence multinucleation, as per Istanbul consensus. ${ }^{21}$

After 12 days of embryo transfer, beta-hCG is checked to confirm pregnancy and the trial medicine is stopped irrespective of the beta-hCG result. LPS will be continued until 10 weeks of gestation if pregnant. A transvaginal ultrasound will be performed 4 weeks after the embryo transfer to confirm viable intrauterine pregnancy.

One of the limitations of our study is that we did not assess serum DHEA along with serum testosterone. As age increases serum DHEA and testosterone level decreases. It is the testosterone that is going to bind to the androgen receptor ${ }^{22}$ and both high and low levels of serum testosterone are detrimental for implantation ${ }^{18} 19$ hence, we chose to monitor serum testosterone over DHEA. Similarly, after initiating progesterone and DHEA, we did not monitor the serum DHEAS levels as DHEA-S and progesterone could cross-react with the commercial immunoassays in a dose-dependent manner resulting in false values. $^{23}$

\section{Sample size calculation}

The sample size is calculated for the primary outcome measure-live birth rate based on our retrospective study of 193 patients which showed beneficial results with DHEA supplementation; with the study intervention, with a power of $80 \%$ and $\alpha$ error of 0.05 , our required sample size is 140 after considering $5 \%$ lost to follow-up and 5\% drop out rate.

\section{Statistical analysis}

A patient could enrol for the trial only once. All statistical analysis will be performed by intention to treat principle. Per protocol analysis will also be conducted. The Pharmacy will have a list of participants with their unique identification code and their allocated treatment planned. Complete data of patient and treatment record is documented in an electronic medical system database. Statistical analysis will be performed using SPSS (V.20). For normally distributed data, the mean and SD will be used to describe data location and dispersion and Student's t-test and $\chi^{2}$ test will be used respectively for continuous and categorical data. For not normally distributed data, Mann-Whitney $\mathrm{U}$ test for continuous variables and McNemar's $\chi^{2}$ test for categorical variables will be considered.

\section{Patient and public involvement}

We do not have a formal patient group, but we have discussed this with many of our patients, incorporated their suggestions and they all uniformly agreed that improvement in live birth rate which is our primary outcome measure is what they are looking for. The participants will be informed which treatment they received after the trial is completed by phone as well as email. Also, the study results will be published in the annual patient information booklet. The DHEA capsules (study group) as well as the placebo capsules (control group), additional testosterone testing, additional personnel work, stationery and other consumables that are not part of the routine clinical care will be funded by the hospital.

\section{Ethics and dissemination}

The use of DHEA has not been associated with any major side effects. It is commonly used in patients with a 
decreased ovarian reserve and a recent study by Çelik $e t a t^{6}$ have shown its beneficial effect in implantation. We will be using DHEA in those who have low serum testosterone only for a limited period of 14 days, hence we expect fewer side effects. The side effects usually reported are mainly androgenic, namely, facial hair growth, acne and rarely deepened voice. In case of any side effects and noncompliance, the patient will be asked to stop the medication and escalated to the principal investigator. This study has been approved by the Clinical Trial Registry of India (trial registered prospectively on 16 June 2020) and reporting will be according to the 2013 Standard Protocol Items: Recommendations for Interventional Trials statement. Any modification of the research protocol will be subjected to an authorisation agreement from the Ethics Committee. All participants will provide written informed consent before being randomised into allocated treatment groups. The participants will be informed about the treatment they received after the completion of the trial.

We are planning to embed our study results in clinical practice and disseminate the findings through peerreviewed journal publications, scientific conferences, social media and patient information booklets. The results obtained through this research may help clinicians in understanding the concept of supplementing DHEA and take a judicious decision. If DHEA is found to be effective, it will be a novel cost-effective and safe strategy for improving the implantation rate.

Acknowledgements The authors would like to thank patients for their input and feedback on this study protocol and thank Mr Tittu Raju for his help in the statistics.

Contributors MAN, MA and KJ were responsible for the conception, design and analytical plan. Critical evaluation was performed by all authors. SwatiS, MAN, $A R, R B, A S$ and SankalpS were responsible for writing the first draft and the final manuscript was read and approved by all authors. There is no potential conflict of interest.

Funding There is no significant financial support or conflict of interest associated with this publication, which could have influenced its outcome. This study is supported by the CRAFT Hospital and Research Centre, Kerala, India. Grant number-CHRC/2020/04/492001. As such this research received no specific grant from any funding agency in the public, commercial or not-for-profit sectors. The Hospital funds are being used for the miscellaneous expenses only, it does not have any role in data analysis or interpretation of data or writing of report or any authority over decision to submit the report for publication.

\section{Competing interests None declared.}

Patient and public involvement Patients and/or the public were involved in the design, or conduct, or reporting, or dissemination plans of this research. Refer to the Methods section for further details.

Patient consent for publication Not applicable.

Provenance and peer review Not commissioned; externally peer reviewed.

Open access This is an open access article distributed in accordance with the Creative Commons Attribution Non Commercial (CC BY-NC 4.0) license, which permits others to distribute, remix, adapt, build upon this work non-commercially, and license their derivative works on different terms, provided the original work is properly cited, appropriate credit is given, any changes made indicated, and the use is non-commercial. See: http://creativecommons.org/licenses/by-nc/4.0/.

Author note I am an author on this submission, have adhered to all editorial policies for submission as described in the Information for Authors, attest to having met all authorship criteria, and disclosed all potential conflicts of interest for inclusion on the title page of the submission. Authorship-form attached as supplementary material.
ORCID iD

Apeksha Sahu http://orcid.org/0000-0002-9372-3907

\section{REFERENCES}

1 Okada $\mathrm{H}$, Tsuzuki T, Murata $\mathrm{H}$. Decidualization of the human endometrium. Reprod Med Biol 2018;17:220-7.

2 Kajihara T, Brosens JJ, Ishihara O. The role of FOXO1 in the decidual transformation of the endometrium and early pregnancy. Med Mol Morphol 2013;46:61-8.

3 Fouany MR, Sharara FI. Is there a role for DHEA supplementation in women with diminished ovarian reserve? J Assist Reprod Genet 2013;30:1239-44.

4 Conneely OM, Lydon JP. Progesterone receptors in reproduction: functional impact of the A and B isoforms. Steroids 2000;65:571-7.

5 Cloke B, Huhtinen K, Fusi L, et al. The androgen and progesterone receptors regulate distinct gene networks and cellular functions in decidualizing endometrium. Endocrinology 2008;149:4462-74.

6 Çelik Önder, Acet M, İmren A, et al. Dhea supplementation improves endometrial HOXA-10 mRNA expression in poor responders. J Turk Ger Gynecol Assoc 2017;18:160-6.

7 Narkwichean A, Maalouf W, Campbell BK, et al. Efficacy of dehydroepiandrosterone to improve ovarian response in women with diminished ovarian reserve: a meta-analysis. Reprod Biol Endocrinol 2013;11:44.

8 Zhang M, Niu W, Wang Y, et al. Dehydroepiandrosterone treatment in women with poor ovarian response undergoing IVF or ICSI: a systematic review and meta-analysis. J Assist Reprod Genet 2016;33:981-91.

9 Gibson DA, Simitsidellis I, Kelepouri O, et al. Dehydroepiandrosterone enhances decidualization in women of advanced reproductive age. Fertil Steril 2018;109:728-34.

10 Gao F, Bian F, Ma X, et al. Control of regional decidualization in implantation: role of FoxM1 downstream of HOXA10 and cyclin D3. Sci Rep 2015;5:13863.

11 Lynch VJ, Brayer K, Gellersen B, et al. HoxA-11 and FOXO1a cooperate to regulate decidual prolactin expression: towards inferring the core transcriptional regulators of decidual genes. PLoS One 2009; 4 :e6845.

12 Shuya LL, Menkhorst EM, Yap J, et al. Leukemia inhibitory factor enhances endometrial stromal cell decidualization in humans and mice. PLoS One 2011;6:e25288.

13 Williams MRI, Dawood T, Ling S, et al. Dehydroepiandrosterone increases endothelial cell proliferation in vitro and improves endothelial function in vivo by mechanisms independent of androgen and estrogen receptors. J Clin Endocrinol Metab 2004;89:4708-15.

14 Lecce L, Lam YT, Lindsay LA, et al. Aging impairs VEGF-mediated, androgen-dependent regulation of angiogenesis. Mol Endocrinol 2014;28:1487-501.

15 Cai J, Hong Y, Weng C, et al. Androgen stimulates endothelial cell proliferation via an androgen receptor/VEGF/cyclin A-mediated mechanism. Am J Physiol Heart Circ Physiol 2011;300:H1210-21.

16 Burger HG. Androgen production in women. Fertil Steril 2002;77:3-5.

17 Gleicher N, Kim A, Weghofer A, et al. Hypoandrogenism in association with diminished functional ovarian reserve. Hum Reprod 2013;28:1084-91.

18 Cermik D, Selam B, Taylor HS. Regulation of HOXA-10 expression by testosterone in vitro and in the endometrium of patients with polycystic ovary syndrome. J Clin Endocrinol Metab 2003;88:238-43.

19 Lu Q, Shen H, Li Y, et al. Low testosterone levels in women with diminished ovarian reserve impair embryo implantation rate: a retrospective case-control study. J Assist Reprod Genet 2014;31:485-91.

20 Hausknecht V, Lopez de la Osa E, Gurpide E. In vitro metabolism of C19 steroids in human endometrium. J Steroid Biochem 1982;17:621-9.

21 Alpha Scientists in Reproductive Medicine and ESHRE Special Interest Group of Embryology. The Istanbul consensus workshop on embryo assessment: proceedings of an expert meeting. Hum Reprod 2011;26, :1270-83.

22 Burger HG. Androgen production in women. Fertil Steril 2002;77 Suppl 4:3-5.

23 Franasiak JM, Thomas S, Ng S, et al. Dehydroepiandrosterone (DHEA) supplementation results in supraphysiologic DHEA-S serum levels and progesterone assay interference that may impact clinical management in IVF. J Assist Reprod Genet 2016;33:387-91 https:// doi.org/10.1007/s10815-016-0650-3 